\title{
Influencia de la anisotropía en la durabilidad de las dolomías Cretácicas de la Comunidad de Madrid frente a la cristalización de sales
}

\section{Influence of anisotropy on the durability of Madrid-region Cretaceous dolostone exposed to salt crystallization processes}

\author{
R. Fort ${ }^{(*)}$, B. Fernández-Revuelta(**), M. J. Varas ${ }^{(*, * * *)}$, M. Álvarez de Buergo ${ }^{(*)}$ y M. Taborda-Duarte(*)
}

Recepción/Received: 16-VI-07

Aceptación/Accepted: 19-IX-07

Publicado online/Online publishing: 18-XII-07

\section{RESUMEN}

En este trabajo se estudia el comportamiento de las dolomías de Redueña y de Torrelaguna frente a los efectos de la cristalización de sales. Ambos materiales han sido ampliamente utilizados en la construcción del Patrimonio arquitectónico y monumental de la región de Madrid. Para la selección de las probetas pétreas a ensayar, se midió la velocidad de propagación de ondas ultrasónicas $(\mathrm{Vp})$ en cerca de un centenar de muestras cúbicas de cada variedad. Se calcularon los índices de anisotropía y se realizó un proceso de clustering para su clasificación en función de niveles de la misma. De cada clase se tomaron dos probetas representativas y se procedió a su caracterización petrográfica y petrofísica antes y después de someterlas a ciclos de cristalización de sales. Para la evaluación del envejecimiento se registró la variación de peso y se documentó el deterioro visual observado. El patrón de degradación para cada uno de los materiales frente a los ciclos es bastante distinto: la dolomía de Redueña es la que presenta un deterioro más intenso con descamaciones y desplacados mientras que la de Torrelaguna tiende a generar fragmentaciones y formas redondeadas.

Palabras clave: dolomías, ultrasonidos, índices de anisotropía, cristalización de sales, deterioro, piedra natural.

\section{SUMMARY}

The behaviour of dolostone exposed to the effects of salt crystallization processes was studied on materials from Redueña and Torrelaguna in the province of Madrid, Spain. Much of the region's architectural and monumental heritage was built with the materials from these two sites. Stone specimens were selected for testing on the basis of ultrasonic wave propagation velocity (Vp) measurements taken in around one hundred cubic samples of each variety. The anisotropy indices were calculated and the samples were clustered on the basis of the results. Two representative samples of each class were taken and characterized for their petrographic and petrophysical properties before and after exposure to salt crystallization cycles. Ageing was evaluated in terms of weight loss and the visual deterioration observed. The pattern of decay found varied substantially in the two materials: the Redueña material exhibited more intense deterioration, with scaling and spalling, while the Torrelaguna dolostone tended to generate fragmentation and rounding at corners and edges.

Keywords: dolostone, ultrasound, anisotropy indices, salt crystallization, deterioration, natural stone.

(*) Instituto de Geología Económica, CSIC-UCM (Madrid, España)

(**) Instituto Geológico y Minero de España, IGME (Madrid, España).

(***) Universidad Complutense de Madrid (Madrid, España). 


\section{INTRODUCCIÓN}

La caracterización de la piedra natural resulta esencial para evaluar la viabilidad de sus posibles usos en construcción, así como para establecer su durabilidad en el tiempo (1). Ruedrich y Siegesmund (2) señalan la dificultad de determinar la calidad de la piedra natural debido al carácter heterogéneo y a la frecuente anisotropía de sus propiedades petrofísicas. La mayoría de los materiales pétreos son anisótropos, es decir, sus propiedades varían según la dirección en que se midan; en ocasiones la anisotropía no es detectable a simple vista y se requiere de medidas en laboratorio (3).

El conocimiento de la anisotropía de los materiales pétreos tiene una influencia directa en la colocación en obra de los elementos, ya que su disposición según una u otra orientación condiciona la forma de trabajar del elemento y su deterioro futuro. Igualmente es un factor a tener en cuenta a la hora de abordar intervenciones de sustitución y reemplazamiento en obras patrimoniales.

Una de las técnicas que posibilita la determinación de la anisotropía de los materiales es la técnica de prospección ultrasónica, basada en la medida de la velocidad de propagación de ondas ultrasónicas. El tiempo de propagación de la onda depende en gran medida de la densidad del material, de su porosidad, de su mineralogía, de su textura y anisotropía, y de su contenido en agua (4). Entre sus principales ventajas figuran la de ser una técnica no destructiva y la de su fácil manejo, permitiendo la toma de una gran número de medidas sin gran esfuerzo en un período de tiempo corto, lo cual posibilita una mejor evaluación de los resultados obtenidos (5). La técnica ultrasónica se utiliza en el estudio de la piedra natural y otros materiales de construcción con diversos propósitos: para la caracterización de los materiales (5-12); para la evaluación y cuantificación de su deterioro $(1,13-17)$; para la evaluación de productos consolidantes $(18,19)$, y para la determinación de la procedencia de los materiales utilizados en construcciones históricas (20-22).

Birch (23) determinó el coeficiente de anisotropía mediante la siguiente ecuación [1]:

$$
\mathrm{k}=\left[\left(V p_{\max }-V p_{\text {min }}\right) / V p_{\text {mean }}\right] \cdot 100
$$

Birch (23) determined the anisotropic coefficient from the following equation [1]:

where $V p_{\max }$ is the maximum, $V p_{\min }$ the minimum and $V p_{\text {mean }}$ the mean $P$-wave velocity, measured multi-directionally. Subsequently, Guydader and Denis (24) determined two anisotropy indices based on the measurement of $P$-wave velocity $(V p)$ in the three spatial directions. These anisotropy indices have been used by many authors (5, $7,9,13,14,17,21,25,26$ working in areas such as construction materials and the conservation of the architectural heritage. Shön (27) suggested the following anisotropy factor [2]: 


$$
A=\left(\left(V p_{\max }-V p_{\min }\right) / V p_{\min }\right) \cdot 100
$$

Ruedrich y Siegesmund (2) utilizan la siguiente fórmula para calcular la anisotropía [3]:

$$
A=\left(\left(V p_{\max }-V p_{\min }\right) / V p_{\max }\right) \cdot 100
$$

Ruedrich and Siegesmund (2) adopted the following formula to calculate anisotropy [3]:

La piedra natural de construcción se deteriora inexorablemente con el tiempo debido a diversos agentes, procesos y mecanismos de degradación. La cristalización de sales solubles en los poros de los materiales pétreos es uno de los principales mecanismos reconocidos como causantes de su deterioro y que compromete enormemente su durabilidad en el tiempo (28-36). El crecimiento de cristales salinos en el interior de los poros de un material rocoso puede generar tal grado de tensiones que superen el umbral de la resistencia a tracción del material, llegando a su completa arenización, incluso reduciéndolo a polvo. Es por ello que la cristalización de sales se contempla como uno de los mecanismos de deterioro en la serie de ensayos de envejecimiento artificial acelerado que se realizan de manera rutinaria y estandarizada en la piedra natural $(37,38)$. Sin embargo, algunos autores ya comienzan a demandar una revisión de este ensayo (39-41), principalmente en lo que al procedimiento del ensayo se refiere. Un aspecto que generalmente no se contempla es la influencia que la anisotropía tiene en el proceso de deterioro de los materiales pétreos, y en concreto en el mecanismo de cristalización de sales $(2,42,43)$.

En este trabajo se propone la combinación de los índices de anisotropía medidos en dos variedades de dolomías, junto con un proceso de clustering, para establecer diferentes clases de anisotropía. Posteriormente se ha pretendido determinar el grado de influencia que la anisotropía de estas dos variedades pétreas puede tener en el deterioro al que van a estar sometidas, y, por tanto, si debería tenerse en cuenta en la realización de ensayos de envejecimiento artificial acelerado.

\section{MATERIALES Y MÉTODOS}

Los materiales pétreos ensayados se han seleccionado por constituir materiales de construcción tradicionales en la región de Madrid. Para esta investigación se ha trabajado con dos variedades dolomíticas del Cretácico Superior, procedentes de canteras inactivas de Redueña y Torrelaguna. Ambos tipos de materiales fueron extensamente utilizados en la construcción del patrimonio arquitectónico y monumental de Madrid capital y de su provincia (44).

Se ha observado que los monumentos y edificios construidos con estas dolomías, sufren con frecuencia procesos de cristalización de sales que contribuyen a su deterioro.

De cada variedad se obtuvo cerca de un centenar de probetas cúbicas $(50 \pm 5 \mathrm{~mm})$. Este tipo de probetas cúbicas
When used in construction, natural stone inescapably deteriorates with time due to the degradational action of a number of agents, processes and mechanisms. The crystallization of soluble salts in the pores of stone materials is one of the main mechanisms acknowledged to induce deterioration, and to severely compromise stone durability (28-36). The growth of saline crystals in the pores of rocky matter can generate stress that exceeds the stone's tensile strength threshold, leading to granular disaggregation and pulverization. For this reason, salt crystallization is included as one of the deterioration mechanisms in the series of standard accelerated ageing tests routinely conducted on natural stone $(37,38)$. Some authors (39-41) have begun to call for a revision of this test, however, primarily in connection with the procedures involved. One aspect that generally goes unnoticed is the effect of anisotropy on the deterioration of stone materials, and specifically on salt crystallization (2, 42, 43).

The present study aimed to establish different types of anisotropy by combining the anisotropy indices measured in two varieties of dolostone limestone with a clustering procedure. This was followed by an attempt to determine the effect of the degree of anisotropy in these two varieties of stone on their deterioration and ascertain whether this property should be taken into account in accelerated ageing tests.

\section{MATERIALS AND METHODS}

The stone materials used for testing were selected because of their role in traditional construction in the region of Madrid. This study focused on varieties of Upper Cretaceous dolostone from abandoned quarries at two sites, Redueña and Torrelaguna. These two materials are found in a fair share of the architectural and monumental heritage in Madrid and its surrounding region (44).

The monuments and buildings erected with these stones are subject to frequent salt crystallization processes that contribute to their deterioration.

Nearly one hundred cubic ( $50 \pm 5 \mathrm{~mm}$ ) specimens were obtained for each type of stone, following RILEM (36) 
es el recomendado por RILEM (36) para la realización de ensayos de durabilidad. Por ello, se utilizaron probetas cúbicas para la realización del envejecimiento por cristalización de sales, y también para la determinación de las propiedades petrofísicas.

La caracterización petrográfica de las láminas delgadas se llevó a cabo mediante un microscopio óptico de luz polarizada Olympus BX 51, con una cámara digital acoplada Olympus DP $12(6 \mathrm{~V} / 2,5 \AA)$, y utilizando un programa Olympus DP-Soft (versión 3.2). Se utilizó la tinción de Alizarin (Red S) para diferenciar calcita de dolomita (45).

La porosidad y la distribución del tamaño de poro se determinaron mediante un porosímetro por intrusión de mercurio Autopore IV 9520, Micromeritics, estableciendo el límite entre micro y macroporosidad en $5 \mu \mathrm{m}$ de diámetro de poro.

El tiempo de propagación de las ondas $\mathrm{P}$ se midió con un equipo PUNDIT CNS Electronics, con una precisión de 0,1 $\mu \mathrm{s}$. Algunas desviaciones respecto a la normativa seguida están relacionadas con la forma y dimensiones de las probetas (cúbica de $50 \pm 5 \mathrm{~mm}$ de lado en vez de las probetas prismáticas que recomienda la norma UNE-EN 14579: 2005 de velocidad de propagación de ultrasonidos (46)), el número mínimo de probetas a ensayar (de 6 se ha aumentado a 8), y la frecuencia de los transductores utilizados, que fue de $1 \mathrm{MHz}$.

Se utilizaron transductores con una superficie de contacto plana de 11,82 mm de diámetro. Para facilitar y asegurar el contacto entre transductores y la superficie de las probetas, se utilizó una pasta de agua y carboximetilcelulosa (Sichozell Kleister, Henkel). Se utilizó el modo de transmisión/recepción directa, midiendo a través de las caras paralelas y opuestas de las probetas cúbicas, en las tres direcciones del espacio. La anisotropía se ha estimado mediante los índices propuestos por Guydader and Denis (24), índices muy utilizados atendiendo a las referencias bibliográficas y que fueron empleados por Zezza en 1990 (7) para determinar las propiedades físico-químicas en materiales pétreos de construcción y de cantera. En este trabajo se han seleccionado dichos índices porque, a diferencia de otros, distinguen entre la anisotropía existente a nivel "planar" o bidimensional y a nivel tridimensional, lo cual, a priori, puede condicionar el tipo de deterioro que se va a generar en un material. Dichos índices son la anisotropía total (dM\%), considerando los tres ejes del espacio, y anisotropía relativa (dm\%), considerando sólo dos de ellos [4] [5]; ambos índices ofrecen información complementaria, ya que cada índice nos indica en qué dirección espacial está concentrada la orientación textural y/o mineralógica que condiciona la anisotropía. recommendations for durability tests. Consequently, these cubic specimens were used for both salt crystallization-induced ageing and to determine the petrophysical properties of the stone.

Thin section petrographic characterization was performed with an Olympus BX 51 polarized light optical microscope, connected to an Olympus DP 12 (6V/2.5A) digital camera. The software used was Olympus DP-Soft (version 3.2). The sections were stained with Alizarin (Red S) to differentiate calcite from dolomite (45).

Porosity and pore size distribution were determined with a Micromeritics Autopore IV 9520 mercury intrusion porosimeter. The division between micro- and macroporosity was set at a pore diameter of $5 \mu \mathrm{m}$.

P-wave propagation time was measured to a precision of $0.1 \mu s$ with a PUNDIT CNS Electronics instrument. The modifications introduced in the standards followed included specimen shape and size (cubic, with a 50 $55-\mathrm{mm}$ sides instead of the prismatic specimens recommended by Spanish and European standard UNE-EN 14579: 2005 on sound speed propagation (46)), the minimum number of specimens tested (increased from six to eight) and the frequency of the transducers used, which was $1 \mathrm{MHz}$.

The flat contact area on these transducers was $11.82 \mathrm{~mm}$ in diameter. The bond between the transducers and the surface of the specimens was secured with a water and carboxymethylcellulose paste (Sichozell Kleister, Henkel). Measurements were taken in direct transmission/reception mode, across opposite parallel sides of the cubic specimens in all three spatial directions. Anisotropy was estimated from the indices proposed by Guydader and Denis (24), routinely used throughout the literature and particularly by Zezza in 1990 (7) to determine the physicalchemical properties of construction and quarry stone. These indices were chosen for the present study because, unlike others, they distinguish between "flat" or twodimensional and three-dimensional anisotropy which may, a priori, condition the type of deterioration generated in a material. The two indices involved, total anisotropy $(d M \%)$, found from the three spatial axes, and relative anisotropy (dm\%), found from only two [4] [5], furnish complementary information, for each indicates the spatial direction in which the textural and/or mineralogical orientation that conditions anisotropy is concentrated.

$$
\begin{aligned}
& \mathrm{dM} \%=\left[1-\left(2 \mathrm{~V}_{1} /\left(\mathrm{V}_{2}+\mathrm{V}_{3}\right)\right)\right] \times 100 \\
& \mathrm{dm} \%=\left[\left(2 \times\left(\mathrm{V}_{2}-\mathrm{V}_{3}\right) /\left(\mathrm{V}_{2}+\mathrm{V}_{3}\right)\right] \times 100\right.
\end{aligned}
$$


Los valores que se obtienen al aplicar estas fórmulas tienen diferente significado para cada tipo de material pétreo: un mismo valor determinado en una caliza o en una pizarra no puede ser interpretado de la misma manera, ya que la historia genética de cada roca imprime un tipo de anisotropía determinado al material. Zezza (7) ofrece una serie de valores determinados en diferentes tipos de materiales pétreos.

Una vez obtenidas las tres velocidades en las tres direcciones ortogonales de la probeta, se asigna V1 a la mínima velocidad, V2 a la máxima, y V3 a la intermedia entre las dos anteriores. Con estos datos las probetas que se van a someter a cristalización de sales se reorientan todas de la misma manera en cuanto anisotropía se refiere, con la dirección de mínima velocidad V1 orientada en la vertical o eje $z$, con objeto de simular lo más posible la realidad, ya que generalmente es como se suelen colocar en obra. Una vez calculados los índices de anisotropía de cada probeta, éstos se representan en un gráfico (dm\%/dM\%). La identificación de los valores extremos y atípicos de la nube de puntos obtenida (y también de las velocidades obtenidas) se llevó a cabo mediante la regla de Chebychev. A continuación, se llevó a cabo un proceso de clustering (k-means clustering según Khan y Ahmad (47)) con el fin de establecer diferentes clases de anisotropía para cada tipo de material. Se trata en esencia de un algoritmo de clasificación automática que puede resumirse en los siguientes pasos: 1) se establece el número de clases ( $\mathrm{k}$ o número de centroides de los clusters) de una manera aleatoria y aplicando el método de ensayo y error, con objeto de obtener un número similar de muestras en cada grupo; 2) se asignan los miembros (valores de los datos) a los clusters; 3) se recalculan los nuevos centroides en función del paso anterior $(2) ; 4$ ) se repiten los pasos 2 y 3 hasta que no se produzcan más cambios en los centroides.

Una vez clasificadas las muestras se seleccionaron 2 probetas de las cuatro clases más importantes y se las sometió al ensayo de envejecimiento acelerado por cristalización de sales (37). En este ensayo cada ciclo consta de una fase de inmersión en sulfato sódico decahidratado al $14 \%$ durante 2 horas $\left(20,0 \pm 0,5^{\circ} \mathrm{C}\right)$ y una fase de secado durante al menos 16 horas a $105 \pm 5^{\circ} \mathrm{C}$. Al final de los ciclos se realizan lavados diarios para eliminar las sales hasta alcanzar una conductividad constante inferior a $20 \mu \mathrm{S} / \mathrm{m}$. La evaluación de los efectos de este ensayo sobre las probetas pétreas se realizó mediante la variación de peso y las formas de deterioro, así como mediante la determinación de la porosidad abierta y absorción de agua a presión atmosférica $(48,49)$ y variación de la velocidad de propagación de ultrasonidos y de los índices de anisotropía.
The values obtained when these formulas are applied have a different meaning for each type of stone material: one and the same value found for limestone and slate cannot be interpreted in the same way, for each rock's genetic history imposes a certain type of anisotropy on the material. Zezza (7) listed a series of values found in different types of stone.

Velocities were obtained in each orthogonal direction for each specimen; the lowest was labelled V1, the highest V2 and the intermediate V3. These data were then used to position all the specimens that were to undergo salt crystallization testing in the same manner, anisotropically speaking: namely, with the minimum velocity (V1) direction forming the vertical or z-axis to simulate reality as closely as possible, for this is how the stone is usually placed in construction. The anisotropy indices for each specimen were found and plotted on a (dm\%/dM\%) graph. Chebychev's rule was applied to identify the extreme and atypical values in the cloud of points (and velocities) obtained. Clustering ( $k$-means clustering as described by Khan and Ahmad (47)) was then performed to establish the different classes of anisotropy for each type of material. In essence, this consists in an automatic classification algorithm whose steps can be summarized as follows: 1) the number of classes ( $k$ or number of cluster centroids) is established at random and by trial and error, to obtain a similar number of samples in each group; 2) members (data values) are assigned to the clusters; 3) new centroids are re-calculated on the basis of the results of the preceding step (2); 4) steps 2 and 3 are reiterated until no more changes are detected in the centroids.
After all the samples were classified, two specimens were chosen from each of the four most relevant classes for salt crystallization-induced accelerated ageing (37). In this test, each cycle consisted of immersion in $14 \%$ decahydrated sodium sulphate for two hours $\left(20.0 \pm 0.5^{\circ} \mathrm{C}\right)$, followed by drying for at least 16 hours at $105 \pm 5^{\circ} \mathrm{C}$. The samples were rinsed daily after the cycles to eliminate all the salts, defined to be when conductivity declined to less than $20 \mu \mathrm{S} / \mathrm{m}$. The effects of this test on the specimens was evaluated on the basis of variation in weight, forms of deterioration, open porosity, water absorption at atmospheric pressure $(48,49)$ and variations in ultrasonic propagation velocity and anisotropy indices. 


\section{RESULTADOS}

La piedra de Redueña es una dolomía con estructura interna masiva y formada por cristales rómbicos de hábito idiomorfo. Presenta variaciones texturales que quedan reflejadas en el dominio de dos tamaños de cristal: se identifican zonas compactadas con microcristales $(15-20 \mu \mathrm{m})$ oscuros, sucios y alterados (núcleos opacos), y zonas menos compactadas con mesocristales (60-150 $\mu \mathrm{m})$, claros, limpios y zonados. En esta última zona existen procesos incipientes de dedolomitización (paso de dolomita a calcita) y una porosidad elevada (20-40\%). La morfología de los poros es bastante regular y su tamaño oscila entre $100-200 \mu \mathrm{m}$ y 7 $\mathrm{mm}$; puntualmente aparece rellena por cemento calcítico.

La dolomía de Torrelaguna también es una dolomía masiva formada por cristales rómbicos de hábito idiomorfo. Puntualmente, presenta bandeados debidos a la conservación de restos de su textura deposicional original (estructuras estromatolíticas). Los cristales son microcristales equigranulares $(<50 \mu \mathrm{m})$ de color oscuro y con pocos núcleos opacos. La porosidad es muy elevada ( $>40 \%$ ), pero es una porosidad rellena en un $95 \%$ por cemento calcítico. Dominan los cementos poiquilotópicos y en mosaico blocky. Se detectan procesos iniciales de dedolomitización. También existen acumulaciones puntuales de geles de oxi-hidróxidos de hierro, responsables de la tonalidad rojiza de esta piedra.

Los valores de anisotropía relativa y total para cada probeta, obtenidos a partir de los valores de velocidad en las tres direcciones del espacio, se han representado en las Figuras 1a y 1c. El proceso de clustering permite estable-

\section{RESULTS}

The Redueña variety is a dolostone with a massive internal structure comprising rhombic, idiomorphic crystals. Its textural variations are reflected in the prevalence of two crystal sizes: compacted zones containing dark, dirty and altered (opaque nuclei) microcrystals (15-20 $\mu \mathrm{m}$ ), and other less compacted zones with light-hued, clean and zonated mesocrystals $(60-150 \mu \mathrm{m})$. Incipient dedolomitization (transition from dolomite to calcite) and high porosity (20-40\%) were observed in the latter zone. Pore morphology was found to be fairly regular and pore size to range from $100-200 \mu \mathrm{m}$ to $7 \mathrm{~mm}$; pores were observed to be sporadically filled with calcitic cement.

The Torrelaguna stone is also a dolostone with a massive internal structure comprising rhombic crystals having an idiomorphic habit. It exhibits sporadic banding due to the conservation of its original depositional texture (stromatolithic structures) and owes its reddish hue to the spotty accumulation of iron oxyhydroxide gels. Very few of its dark, equigranular ( $<50 \mu \mathrm{m})$ crystals have opaque nuclei. Although the stone was found to be very porous (>40\%), $95 \%$ of the pores were filled with calcitic cement. Poikilotopic and blocky mosaic cements prevailed. Initial de-dolomitization was detected.

The relative and total anisotropy values obtained for each specimen from the velocity values in the three spatial directions are shown in Figures $1 a$ and $1 c$. The clustering procedure yielded five classes, $I-V$, in ascending order of

Tabla 1 / Table 1

Anisotropía relativa ( $\mathrm{dm} \%)$ y total ( $\mathrm{dM} \%)$ de los clusters establecidos, y velocidad media. Relative $(d m \%)$ and total anisotropy ( $d M \%)$ and mean velocity by cluster

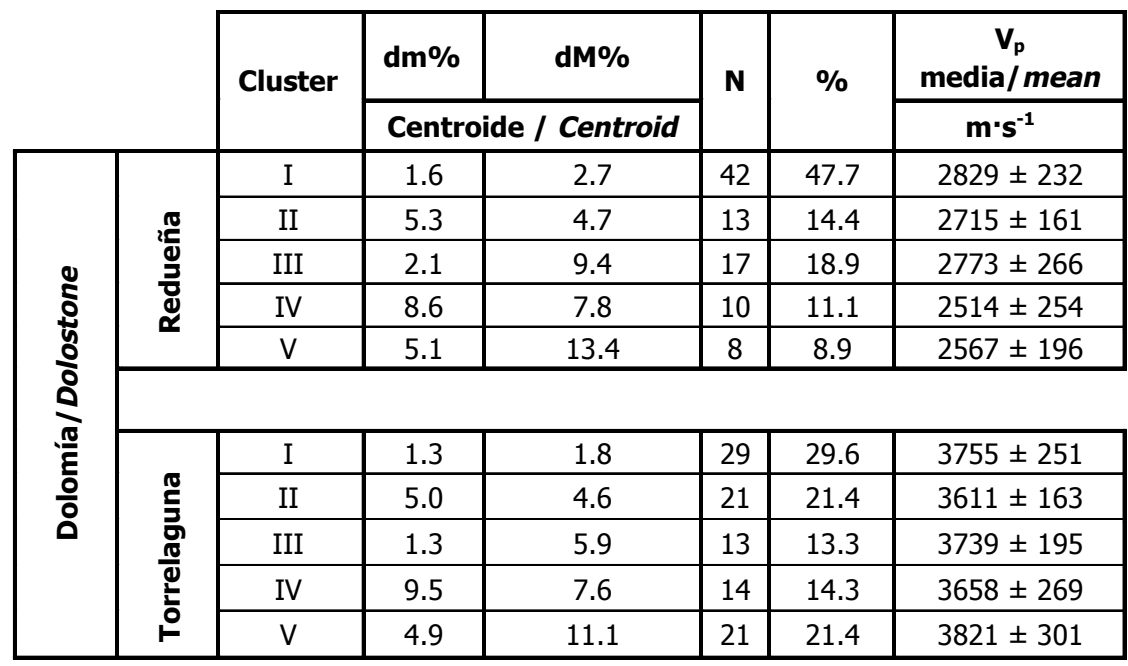

$\mathrm{N}=$ número de elementos, miembros de cada cluster/number of elements in each cluster $\%$ porcentaje de miembros respecto del total/percentage of total 


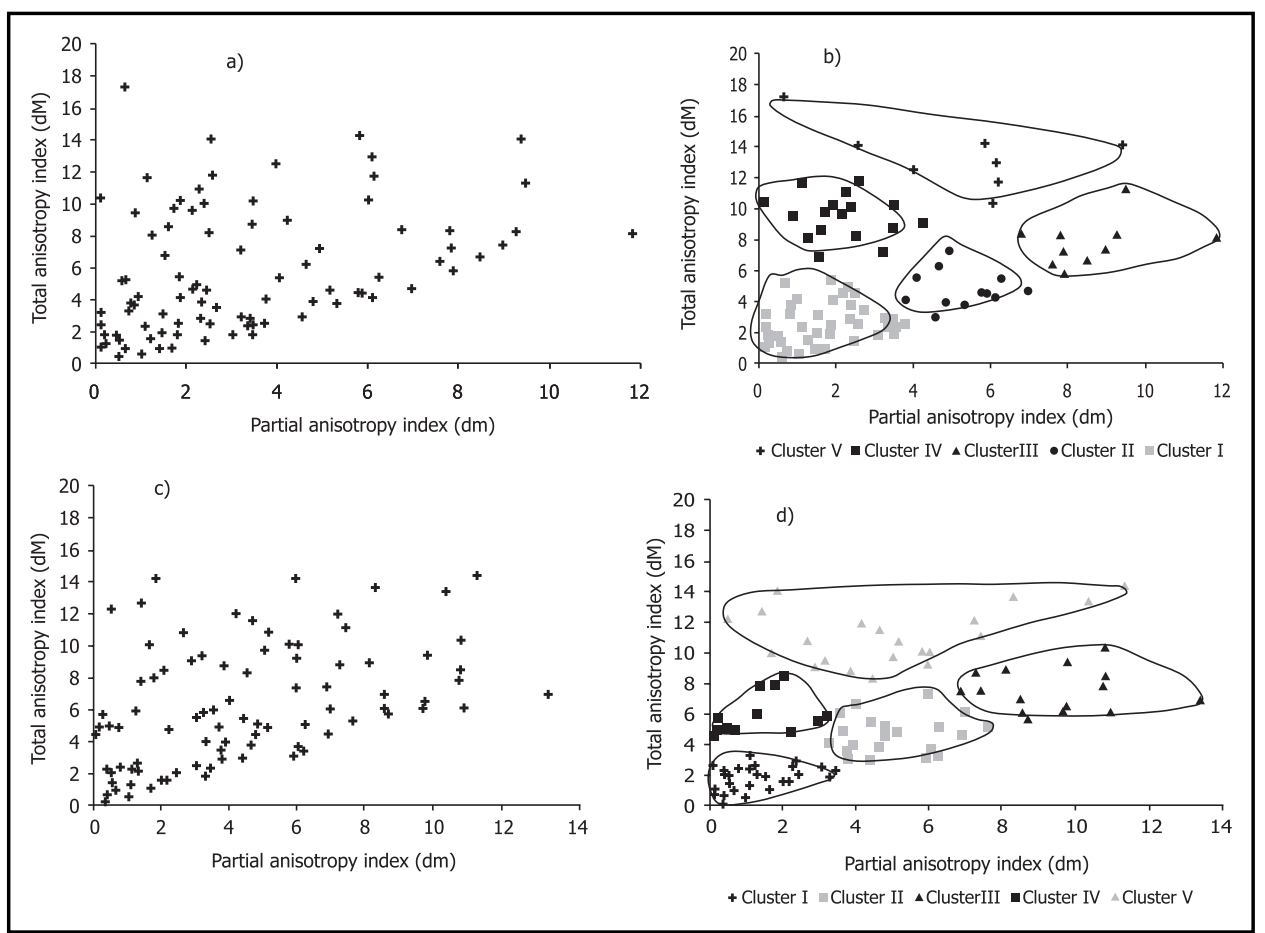

Figura 1. Representación de la nube de puntos correspondiente a los valores de anisotropia relativa y total de las probetas de dolomía de Redueña (a) y de Torrrelaguna (c). Los gráficos b) y

d) muestran las diferentes clases establecidas después de aplicar el proceso de clustering

Figure 1. Relative and total anisotropy values for the Redueña (a) and Torrelaguna (c) dolostone specimens. Cloud of points Graphs b) and d) show the clusters defined.

cer 5 clases, I-V, de menor a mayor anisotropía, para ambos tipos de dolomías (Figuras 1b, 1d). La Tabla 1 muestra los valores de anisotropía relativa y total para cada uno de los clusters establecidos, el número de miembros y su porcentaje respecto al total, así como el valor medio de $\mathrm{Vp}$ para cada cluster.

Para ambos tipos de dolomías, se seleccionaron dos probetas de cada una de las cuatro clases más representativas de cada variedad pétrea para realizar el estudio. La Tabla 2 muestra, entre otros, los valores medios de la ani- anisotropy, for the two dolostones (Figures 1b, 1d). Table 1 gives the relative and total anisotropy values for each of the clusters, along with the number of members, percentage of the total and the mean value of $\mathrm{Vp}$ for each.

Two specimens from each of the four most representative classes of the two stones were selected for testing. Table 2 shows, among others, the mean values for relative and total anisotropy for each dolostone; Table 3 in turn gives

Tabla 2 / Table 2

Propiedades petrofísicas de las dos variedades de dolomías Petrophysical properties of two varieties of dolostone

\begin{tabular}{|c|c|c|}
\hline & \multicolumn{2}{|c|}{$\begin{array}{l}\text { Dolomías del Cretácico Superior/ } \\
\text { Upper Cretaceous dolostones }\end{array}$} \\
\hline & Redueña & Torrelaguna \\
\hline Densidad aparente/ Bulk density $\left(\mathrm{Kg}^{\prime} \mathrm{m}^{-3}\right)$ & $2346 \pm 91$ & $2527 \pm 37$ \\
\hline Absorción de agua/ Water absorption (\%) & $5.6 \pm 1.41$ & $3.4 \pm 0.6$ \\
\hline Porosidad abierta/Open porosity $(\%)$ & $16.4 \pm 3.4$ & $10.1 \pm 1.4$ \\
\hline Velocidad ultrasonidos/Ultrasonic velocity, $\mathrm{Vp}(\mathrm{cm} / \mathrm{s})$ & $2739 \pm 326$ & $3788 \pm 320$ \\
\hline Anisotropía relativa/ Relative anisotropy (dm \%) & $4.26 \pm 3.59$ & $3.58 \pm 3.70$ \\
\hline Anisotropía total/ Total anisotropy (dM\%) & $5.89 \pm 3.66$ & $6.01 \pm 4.14$ \\
\hline
\end{tabular}


Tabla 3 / Table 3

Valores de velocidad de ultrasonidos e índices de anisotropía de las probetas de ambos tipos de dolomías antes y después de los ciclos de cristalización de sales

Ultrasonic velocity values and anisotropy indices for two types of dolostone, before and after salt crystallization testing

\begin{tabular}{|c|c|c|c|c|c|c|c|c|c|}
\hline REDUEÑA & \multirow{2}{*}{$\begin{array}{c}\text { Cluster } \\
\text { anisotropía/ } \\
\text { Anisotropy cluster }\end{array}$} & \multirow{2}{*}{$\begin{array}{l}\text { A* } \\
\text { D** }\end{array}$} & \multirow{2}{*}{$\begin{array}{l}\mathrm{V}_{1} \\
\mathrm{~m} / \mathrm{s}\end{array}$} & \multirow{2}{*}{$\begin{array}{l}\mathrm{v}_{2} \\
\mathrm{~m} / \mathrm{s}\end{array}$} & \multirow{2}{*}{$\begin{array}{l}\mathrm{V}_{3} \\
\mathrm{~m} / \mathrm{s}\end{array}$} & \multirow{2}{*}{$\begin{array}{c}\text { Vp media/ } \\
\text { Mean Vp } \\
\mathrm{m} / \mathrm{s}\end{array}$} & \multirow{2}{*}{$\begin{array}{c}\Delta \mathbf{V p} \\
\%\end{array}$} & \multirow{2}{*}{$\begin{array}{l}\mathrm{dM} \\
\%\end{array}$} & \multirow{2}{*}{$\underset{\%}{\mathrm{dm}}$} \\
\hline $\begin{array}{l}\text { no probeta/ } \\
\text { specimen \# }\end{array}$ & & & & & & & & & \\
\hline \multirow{2}{*}{21} & \multirow{4}{*}{ I } & \multirow{2}{*}{ A } & 2775 & 2824 & 2808 & 2802 & \multirow{2}{*}{-45} & 1.46 & 0.57 \\
\hline & & & 1369 & 1652 & 1636 & 1552 & & 16.73 & 0.97 \\
\hline \multirow{2}{*}{22} & & \multirow{2}{*}{ D } & 2352 & 2411 & 2401 & 2388 & \multirow{2}{*}{-45} & 2.24 & 0.42 \\
\hline & & & 1161 & 1421 & 1334 & 1305 & & 15.72 & 6.32 \\
\hline \multirow{2}{*}{2} & \multirow{4}{*}{ II } & \multirow{2}{*}{ A } & 2644 & 2791 & 2776 & 2737 & \multirow{2}{*}{-62} & 5.01 & 0.54 \\
\hline & & & 1001 & 1075 & 1071 & 1049 & & 6.71 & 0.37 \\
\hline \multirow{2}{*}{6} & & \multirow{2}{*}{ D } & 2461 & 2657 & 2533 & 2551 & \multirow{2}{*}{-45} & 5.16 & 4.78 \\
\hline & & & 1367 & 1430 & 1394 & 1397 & & 3.19 & 2.55 \\
\hline \multirow{2}{*}{27} & \multirow{4}{*}{ III } & \multirow{4}{*}{ D } & 2367 & 2612 & 2559 & 2512 & \multirow{2}{*}{-12} & 8.45 & 2.05 \\
\hline & & & 1710 & 2498 & 2453 & 2220 & & 30.92 & 1.82 \\
\hline \multirow{2}{*}{14} & & & 2521 & 2620 & 2344 & 2495 & \multirow{2}{*}{-67} & -1.57 & 11.12 \\
\hline & & & 712 & 941 & 800 & 818 & & 18.21 & 16.20 \\
\hline \multirow{2}{*}{9} & \multirow{4}{*}{ IV } & \multirow{4}{*}{ D } & 2303 & 2688 & 2495 & 2495 & \multirow{2}{*}{-34} & 11.13 & 7.45 \\
\hline & & & 1519 & 1790 & 1596 & 1635 & & 10.28 & 11.46 \\
\hline \multirow{2}{*}{4} & & & 2227 & 2496 & 2264 & 2329 & \multirow{2}{*}{-46} & 6.43 & 9.75 \\
\hline & & & 1080 & 1379 & 1333 & 1264 & & 20.35 & 3.39 \\
\hline
\end{tabular}

\begin{tabular}{|c|c|c|c|c|c|c|c|c|c|}
\hline TORRELAGUNA & & & & & & Vp media/ & & $d M$ & dm \\
\hline $\begin{array}{l}\text { no probeta/ } \\
\text { specimen \# }\end{array}$ & $\begin{array}{c}\text { anisotropía/ } \\
\text { Anisotropy cluster }\end{array}$ & $\mathrm{D} * *$ & $\mathrm{~m} / \mathrm{s}$ & $\mathrm{m} / \mathrm{s}$ & $\mathrm{m} / \mathrm{s}$ & $\begin{array}{c}\text { Mean Vp } \\
\mathrm{m} / \mathrm{s}\end{array}$ & $\%$ & $\%$ & $\%$ \\
\hline \multirow{2}{*}{9} & \multirow{4}{*}{ I } & A & 3382 & 3583 & 3507 & 3491 & \multirow{2}{*}{-20} & 4.60 & 2.14 \\
\hline & & D & 2765 & 2879 & 2766 & 2803 & & 2.04 & 4.00 \\
\hline \multirow{2}{*}{10} & & A & 3553 & 3675 & 3566 & 3598 & \multirow{2}{*}{-23} & 1.86 & 3.01 \\
\hline & & D & 2654 & 2970 & 2741 & 2788 & & 7.06 & 8.02 \\
\hline \multirow{2}{*}{21} & \multirow{4}{*}{ II } & $A$ & 3558 & 3929 & 3740 & 3743 & \multirow{2}{*}{-12} & 7.21 & 4.93 \\
\hline & & $\mathrm{D}$ & 3121 & 3524 & 3292 & 3312 & & 8.42 & 6.81 \\
\hline \multirow{2}{*}{30} & & A & 4063 & 4332 & 4107 & 4167 & \multirow{2}{*}{-13} & 3.71 & 5.33 \\
\hline & & $\mathrm{D}$ & 3464 & 3819 & 3579 & 3621 & & 6.35 & 6.49 \\
\hline \multirow{2}{*}{20} & \multirow{4}{*}{ III } & A & 3269 & 3463 & 3439 & 3390 & \multirow{2}{*}{-19} & 5.27 & 0.70 \\
\hline & & $\mathrm{D}$ & 2399 & 2925 & 2916 & 2746 & & 17.86 & 0.31 \\
\hline \multirow{2}{*}{22} & & A & 3694 & 3875 & 3838 & 3802 & \multirow{2}{*}{-14} & 4.21 & 0.96 \\
\hline & & D & 3149 & 3380 & 3253 & 3260 & & 5.05 & 3.83 \\
\hline \multirow{2}{*}{13} & \multirow{4}{*}{ V } & $A$ & 3194 & 3833 & 3616 & 3548 & \multirow{2}{*}{-14} & 14.24 & 5.83 \\
\hline & & $\mathrm{D}$ & 2846 & 3367 & 2936 & 3050 & & 9.69 & 13.68 \\
\hline \multirow{2}{*}{14} & & A & 3258 & 3656 & 3503 & 3472 & \multirow{2}{*}{-29} & 8.98 & 4.27 \\
\hline & & $D$ & 2179 & 2755 & 2497 & 2477 & & 17.02 & 9.82 \\
\hline
\end{tabular}

$A^{*}$ antes de los ciclos de cristalización de sales/Before salt crystalization testing.

D** después de los ciclos de cristalización de sales/After salt crystalization testing.

sotropía relativa y de la anisotropía total medida para cada tipo de dolomía; en la Tabla 3 se han expresado los valores de las tres velocidades medidas en cada una de the values of the three velocities measured in each specimen and the mean, along with relative and total anisotropy before and after ageing, likewise for both varieties. The 
las probetas, la velocidad media, la anisotropía relativa y la total, antes y después del envejecimiento, para cada una de las variedades dolomíticas. $\Delta \mathrm{Vp}$ representa la variación en $\%$ de la velocidad de las ondas $\mathrm{P}$ determinada antes y después del envejecimiento.

En la Tabla 4 se ha expresado la valoración del envejecimiento por cristalización de sales mediante la determinación de la variación de peso, de la absorción de agua y de la porosidad abierta o accesible al agua. symbol $\Delta V p$ represents the per cent variation in P-wave velocity before and after ageing.

Table 4 contains the assessment of salt crystallization ageing based on variations in weight, water absorption and open or porosity accesible to water.

Tabla 4 / Table 4

Evaluación del ensayo de cristalización de sales en las probetas de dolomías mediante la variación de peso, de absorción de agua y de porosidad abierta

Weight loss, water absorption and open porosity in dolostone specimens

before and after salt crystallization testing

\begin{tabular}{|c|c|c|c|c|c|c|}
\hline \multicolumn{2}{|c|}{ REDUEÑA } & \multicolumn{2}{c|}{ ANTES / BEFORE } & \multicolumn{2}{c|}{ DESPUÉS / AFTER } \\
\hline $\begin{array}{c}\text { no probeta/ } \\
\text { specimen \# }\end{array}$ & $\begin{array}{c}\text { Cluster } \\
\text { anisotropía/ } \\
\text { Anisotropy } \\
\text { cluster }\end{array}$ & $\begin{array}{c}\Delta \text { peso/ } \\
\text { weight } \\
\%\end{array}$ & $\begin{array}{c}\text { Absorción } \\
\text { agua/ } \\
\text { Water } \\
\text { absorption \% }\end{array}$ & $\begin{array}{c}\text { Porosidad } \\
\text { abierta/ } \\
\text { Open porosity } \\
\%\end{array}$ & $\begin{array}{c}\text { Absorción } \\
\text { agua/ } \\
\text { Water } \\
\text { absorption \% }\end{array}$ & $\begin{array}{c}\text { Porosidad } \\
\text { abierta/ } \\
\text { Open porosity } \\
\%\end{array}$ \\
\hline 21 & I & -6.04 & 4.3 & 13.3 & 5.6 & 16.1 \\
\hline 22 & I & -22.06 & 8.2 & 22.3 & 10.6 & 26.0 \\
\hline 2 & II & -18.59 & 5.0 & 15.6 & 6.4 & 18.3 \\
\hline 6 & II & -8.54 & 5.2 & 15.4 & 7.0 & 19.0 \\
\hline 27 & III & -4.41 & 4.7 & 14.3 & 5.1 & 15.2 \\
\hline 14 & III & -32.00 & 8.5 & 22.6 & 10.8 & 26.5 \\
\hline 4 & IV & -5.21 & 5.7 & 17.4 & 6.9 & 19.5 \\
\hline
\end{tabular}

\begin{tabular}{|c|c|c|c|c|c|c|}
\hline \multicolumn{2}{|c|}{ TORRELAGUNA } & \multicolumn{2}{c|}{ ANTES / BEFORE } & \multicolumn{2}{c|}{ DESPUÉS / AFTER } \\
\hline $\begin{array}{c}\text { no probeta/ } \\
\text { specimen \# }\end{array}$ & $\begin{array}{c}\text { Cluster } \\
\text { anisotropía/ } \\
\text { Anisotropy } \\
\text { cluster }\end{array}$ & $\begin{array}{c}\Delta \text { peso/ } \\
\text { weight } \\
\%\end{array}$ & $\begin{array}{c}\text { Absorción } \\
\text { agua/ } \\
\text { Water } \\
\text { absorption \% }\end{array}$ & $\begin{array}{c}\text { Porosidad } \\
\text { abierta/ } \\
\text { Open porosity } \\
\%\end{array}$ & $\begin{array}{c}\text { Absorción } \\
\text { agua/ } \\
\text { Water } \\
\text { absorption \% }\end{array}$ & $\begin{array}{c}\text { Porosidad } \\
\text { abierta/ } \\
\text { Open porosity } \\
\%\end{array}$ \\
\hline 9 & I & -15.84 & 3.4 & 10 & 3.8 & 10.9 \\
\hline 10 & I & -10.84 & 3.4 & 10.1 & 3.6 & 11.0 \\
\hline 21 & II & -4.65 & 2.9 & 8.8 & 3.2 & 9.1 \\
\hline 30 & II & -5.21 & 2.8 & 8.6 & 2.9 & 9.1 \\
\hline 20 & III & -12.58 & 3.3 & 9.8 & 3.6 & 10.7 \\
\hline 22 & III & -7.67 & 3.7 & 10.9 & 3.7 & 11.2 \\
\hline 13 & V & -33.38 & 4.3 & 12.4 & 4.5 & 12.3 \\
\hline 14 & V & -15.49 & 3.4 & 10.4 & 3.8 & 11.5 \\
\hline
\end{tabular}

En cuanto al ensayo de cristalización de sales, las probetas de Torrelaguna se sometieron a los 15 ciclos de cristalización de sales que indica la norma. Las probetas de dolomía de Redueña, sin embargo, sólo se sometieron a 7 ciclos, ya que algunas de las probetas alcanzaron un grado de deterioro muy alto lo que imposibilitaba la continuidad del ensayo ante el riesgo de la destrucción total que impediría la realización de ensayos de comparación entre el inicio y el final del ensayo.
The 15 salt crystallization cycles specified in the standard were run on the Torrelaguna specimens. The Redueña dolostone, however, was only subjected to seven cycles, for some of the specimens became so severely deteriorated that the test had to be interrupted in light of the risk of total destruction, which would have ruled out any comparison between initial and end test results. 
Respecto al deterioro visual observado después del envejecimiento (Figuras 2 y 3), se observan morfologías de deterioro similares a las definidas por otros autores en calizas (50). En las probetas de la dolomía de Redueña se observa una pérdida de volumen fundamentalmente de los vértices y aristas de los cubos pétreos (Figuras 3a) entre 3,0 y 8,0 $\mathrm{mm}$ desde la superficie. La cara que sufre mayor deterioro es la superior, la opuesta a la cara de apoyo durante el ensayo de cristalización, que llega a redondearse hasta alcanzar unos $37 \mathrm{~mm}$ de diámetro. Este mayor deterioro concentrado en la cara superior está favorecido por las propias condiciones y características del ensayo. En los casos de deterioro más pronunciado aparecen grietas perpendiculares a esta cara superior que se transforman en placas (Figura 3a) (desde 3-5 mm de espesor en el centro de la cara hasta 8-12 mm en los bordes). Cada placa (Figura 3b) está formada por una cubierta endurecida y compacta de 1.5-2 mm. Hasta los $12 \mathrm{~mm}$ de profundidad de la superficie, la placa está constituida por escamas (Figura 3b). El máximo deterioro se produce cuando la cara superior se erosiona, y las placas se separan de las caras (desplacado). Lo que queda de la probeta (Figura 3c) se encuentra arenizado y pulverulento. Las caras laterales también quedan redondeadas, con un diámetro entre 35 y $40 \mathrm{~mm}$. La cara
The visual deterioration observed after ageing (Figures 2 and 3) consisted in morphologies similar to those defined in limestone by other authors (50). The Redueña specimens showed dimensional losses essentially around the vertices and edges of the stone cubes (Figure 3a), ranging from 3.0 to $8.0 \mathrm{~mm}$ in depth. The top, i.e., the side opposite the side on which the cubes rested during the crystallization test, exhibited the severest deterioration, rounding down to a final size of about $37 \mathrm{~mm}$ in diameter. This top-side concentration of deterioration was favoured by test conditions and characteristics. Where deterioration was most intense, cracks perpendicular to this side appeared, which developed into loose membranes (Figure 3a) (from 3-5 $\mathrm{mm}$ thick in the centre to 8-12 $\mathrm{mm}$ thick along the edges). Each membrane (Figure 3b) had a hard, compact cover 1.5-2 mm deep and scales to a depth of 12 $\mathrm{mm}$ from the surface (Figure $3 \mathrm{~b}$ ). Maximum deterioration occurred when the top eroded and the patches worked loose from the surface (spalling). What little was left of the specimen (Figure 3c) was disaggregated and powdery. The lateral faces were also rounded, with diameters ranging from 35 to $40 \mathrm{~mm}$. The edges also wore away around

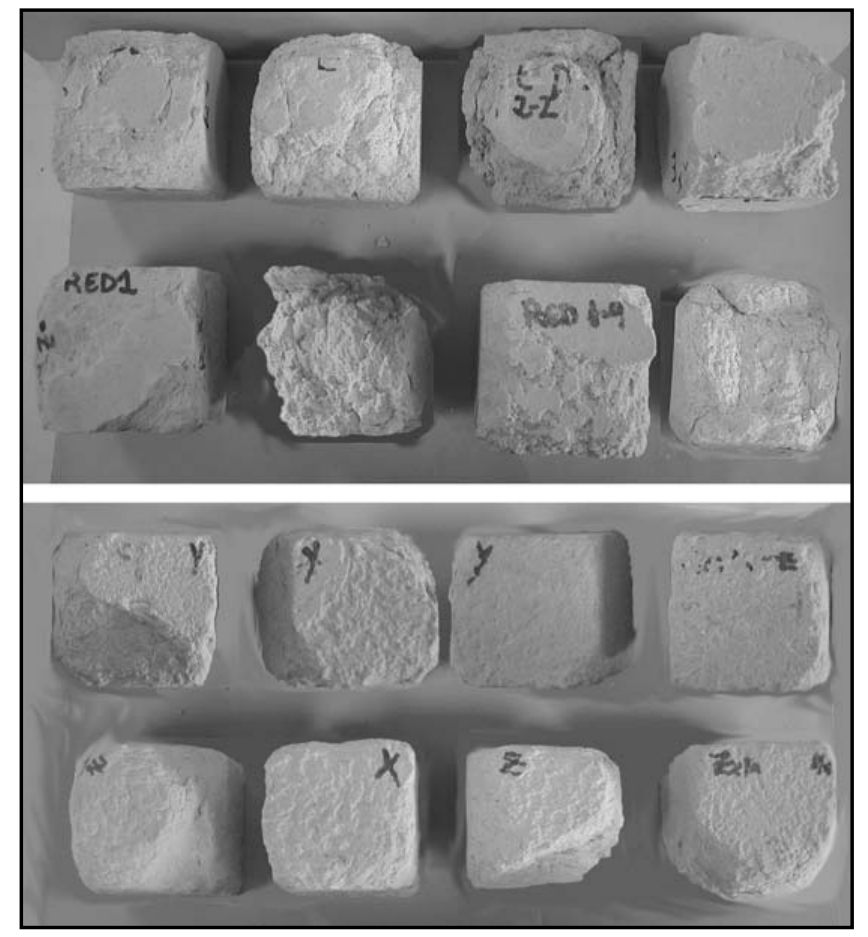

Figura 2. Aspecto de las probetas después del ensayo de cristalización de sales (lado de los cubos $\sim 50 \mathrm{~mm}$. Imagen superior: dolomía de Redueña; imagen inferior: dolomía de Torrelaguna. En cada imagen, las dos probetas de la esquina superior izquierda pertenecen al grupo de menor anisotropia, mientras que las dos probetas de la esquina inferior derecha, al grupo de máxima anisotropía.

Figure 2. Specimens after the salt crystallization test (cube dimension $\sim 50 \mathrm{~mm}$ ). Above: Redueña dolostone; below: Torrelaguna dolostone. The two specimens on the upper left of each photograph pertained to the lowest, and the two samples on the lower right to the highest anisotropy group. 
inferior de apoyo también se redondea (47 mm de diámetro), sin presentar placa paralela a su superficie

En las probetas de la dolomía de Torrelaguna (Figura 2) se produce una pérdida masiva de material pétreo en torno a los vértices y aristas, especialmente de la cara superior (Figura 3d), en forma de fragmentaciones. La parte superior de los cubos se redondea con tendencia a crear un bolo. Este tipo de deterioro lo define la presencia de múltiples fisuras concéntricas, a modo de piel de cebolla. La superficie de las caras laterales y verticales, se endurece (2 $\mathrm{mm}$ de espesor); bajo esta superficie endurecida, se localiza un espesor de descamaciones de 13-17 mm (Figura 3e). El aspecto del núcleo del cubo es rugoso y compacto. En los casos de deterioro más extremo, la superficie de la cara superior se erosiona de tal manera que la altura de las probetas cúbicas se reduce hasta alcanzar los $45-35 \mathrm{~mm}$ (Figura 3f). Las caras laterales verticales y la inferior de apoyo, también se redondean. Sin embargo, lo que queda de la probeta sigue siendo compacto y duro. Varias probetas han experimentado la pérdida total de un vértice (Figura 2), lo cual ha sido responsable de la importante pérdida de peso registrada. the bottom (47 mm in diameter), although no loosened patches were observed parallel to the surface.

The Torrelaguna specimens (Figure 2) underwent massive loss of material around vertices and edges, particularly at the top (Figure 3d), as a result of fragmentation. The top of the cubes tended to round into boulder-like formations. This type of deterioration was characterized by the presence of multiple concentric cracks, resembling onion skin. Scaling to depths of 13-17 mm was observed under the hardened surface ( $2 \mathrm{~mm}$ thick) on the vertical sides (Figure 3e). The core of the cube was compact and rough. Where deterioration was most intense, the top was so eroded that the height of the cubic specimens declined to 45-35 mm (Figure 3f). The lateral and bottom faces were also found to deteriorate around the edges, leaving a round core. Nonetheless, what was left of the specimen was hard and compact. Several specimens lost at least one vertex altogether (Figure 2), which would explain the substantial weight loss recorded.

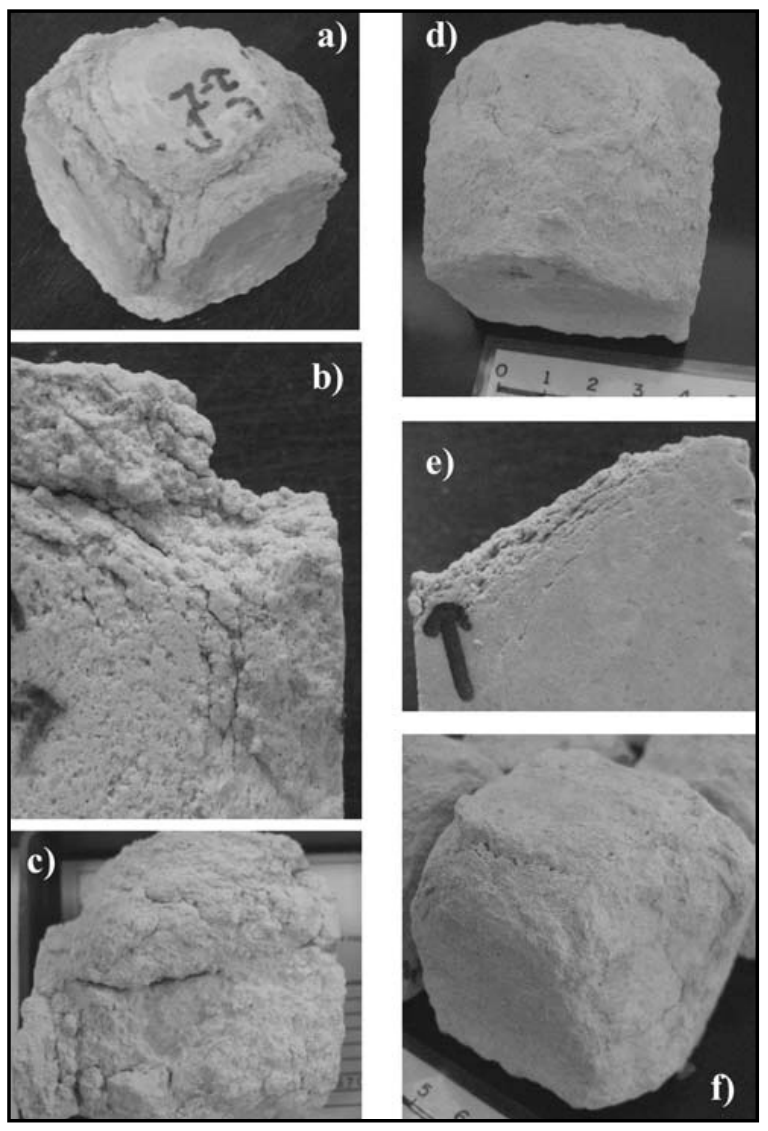

Figura 3. Aspecto de las probetas de dolomía después de los cielos de cristalización de sales. a-c) Dolomía de Redueña; d-f) Dolomía de Torrelaguna.

Figure 3. Dolostone specimens after the salt crystallization cycles: a-c) Redueña dolomite; $d-f$ ) Torrelaguna dolomite. 
En la Figura 4 se ha representado la distribución porosimétrica, para cada dolomía, de la piedra antes del envejecimiento por cristalización de sales, y después, diferenciando entre una muestra de alta y de baja anisotropía.
Figure 4 shows the pore size distribution for high and low anisotropy samples of each dolostone before and after salt crystallization ageing.

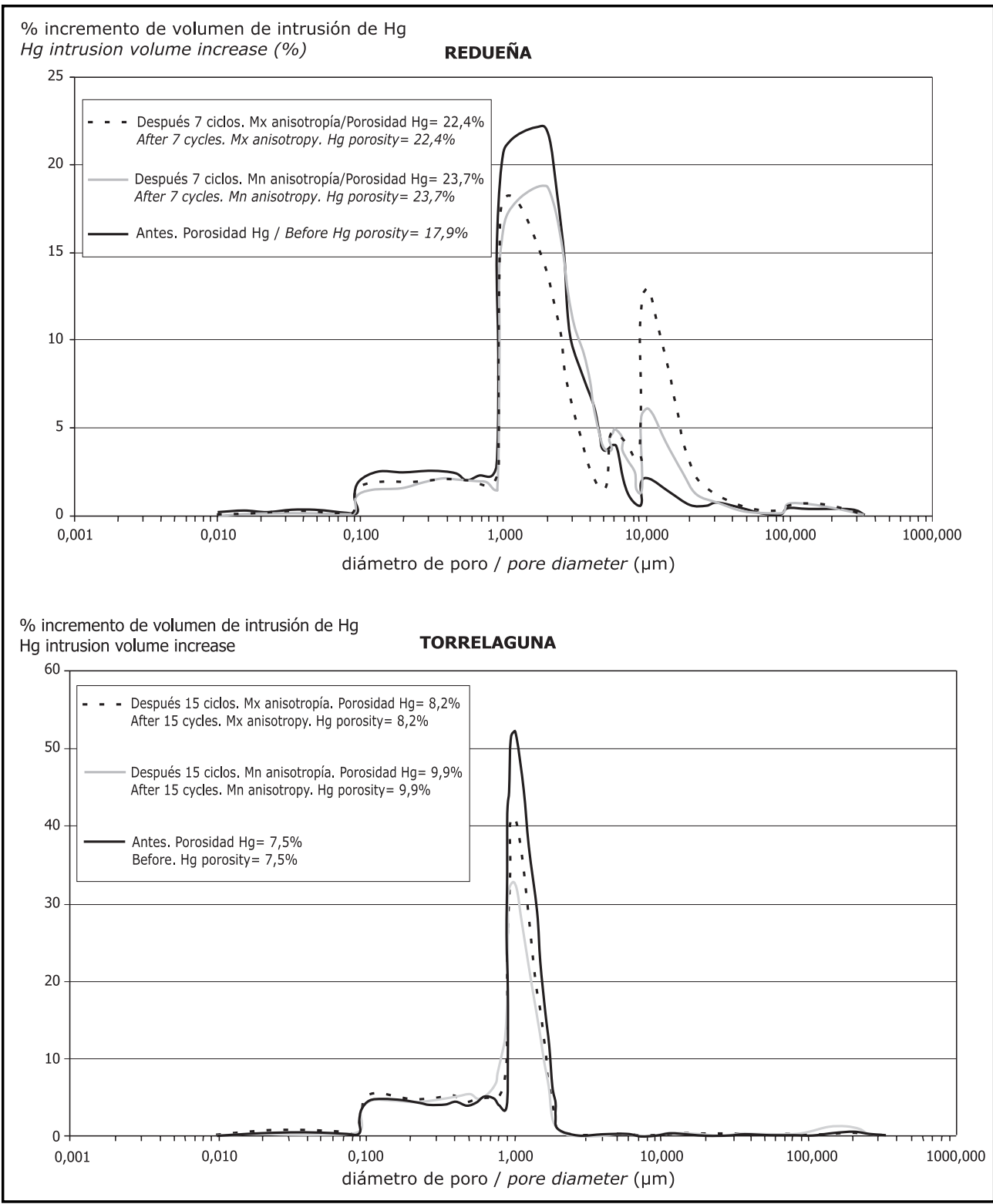

Figura 4. Curvas de distribución de tamaño de poro. En cada uno de los gráficos de ambos tipos de dolomias, se compara la curva de una muestra antes de los cielos de cristalización (línea negra continua) con las curvas de dos muestras después de los ciclos, una de ellas la de máxima anisotropía (linea discontinua) y la otra la de mínima (linea gris continua).

Figure 4. Pore size distribution curves. Both graphs compare the curve found for a single sample measured prior to the crystallization cycles (solid black) to the curves for two samples measured after the cycles, one for the specimen with the lowest anisotropy (dashes) and the other for the stone with the highest anisotropy (solid grey line).

\section{DISCUSIÓN DE RESULTADOS}

La dolomía de Torrelaguna es una piedra de calidad superior a la de Redueña, con una mayor densidad, menor

\section{DISCUSSION}

The Torrelaguna dolostone exhibited higher quality than the Redueña material, with greater density, lower water 
absorción de agua y porosidad abierta, y una velocidad de ultrasonidos más elevada (Tabla 2). Los valores de anisotropía son moderados-bajos para ambas variedades pétreas y presentan gran dispersión de resultados, como puede también deducirse de la Tabla 1, en la que se ofrecen los valores de anisotropía para cada uno de los clusters establecidos. En Redueña parece que se produce una tendencia a que la velocidad de ultrasonidos disminuya al aumentar la anisotropía. Dicha tendencia no se observa en Torrelaguna. La Figura 1 y la Tabla 1 muestran que los clusters con más miembros son aquéllos de menor anisotropía, especialmente visible para el caso de Redueña, en donde casi el $48 \%$ de todos los elementos de Redueña (probetas medidas), pertenecen al grupo I; en Torrelaguna, los clusters I y II acumulan el $51 \%$ de las probetas.

En general, los valores más altos de anisotropía se tienen para la anisotropía total, lo cual indica que la piedra estudiada tiene una anisotropía más marcada en una de las tres direcciones del espacio y que podría deberse a una ligera orientación laminar, poco marcada, ya que no es apreciable a simple vista.

Las velocidades sónicas medidas después de los ciclos de cristalización de sales, manifiestan un claro descenso de los valores registrados, mucho más elevada la reducción para la dolomía de Redueña (hasta un 67\%) que para la de Torrelaguna (hasta un $29 \%$ como máximo). Las probetas que tienen una mayor disminución de la velocidad de propagación de ultrasonidos presentan un mayor deterioro que se aprecia en mayores incrementos de anisotropía y se producen en probetas que pertenecen al cluster I o de menor anisotropía. Este hecho podría interpretarse como que el deterioro de materiales inicialmente poco anisótropos, les lleva a convertirse en materiales más anisótropos. Los valores de anisotropía relativa $(\mathrm{dm})$ no ofrecen un comportamiento tan claro como los de anisotropía total. En el caso de la cristalización de sales, como se ha visto para esta piedra en la Figura 3, se generan unas descamaciones, fisuras y desplacaciones paralelas a la superficie de la cara de la probeta, que serían las responsables del incremento de esa anisotropía.

En el caso de la dolomía de Torrelaguna, parece que mantiene la misma tendencia de aumento del índice de anisotropía total $\mathrm{dM}$, aunque con incrementos menores, y en este caso, a diferencia de Redueña, es difícil asignar que los mayores incrementos se produzcan en probetas con una anisotropía inicial más baja, al producirse más dispersión de resultados. Lo que sufre este tipo de piedra es un incremento del índice de anisotropía relativa, aspecto que no se constataba en la piedra de Redueña.

La pérdida de peso experimentada por ambas dolomías es muy superior para la variedad de Redueña, especialmente teniendo en cuenta que esta dolomía experimentó 7 absorption and open porosity and a higher ultrasonic velocity (Table 2). The anisotropy values were moderate to low for both varieties of stone, although the results showed considerable scatter, as may be deduced from the anisotropy values for the various clusters given in Table 1. Ultrasonic velocity seemed to decline with rising anisotropy in the Redueña material, but not in the Torrelaguna variety. Figure 1 and Table 1 show that the lowest anisotropy values were recorded for the most densely populated clusters, a finding particularly visible in the case of Redueña, where nearly $48 \%$ of all the specimens measured were in Group I; in Torrelaguna, clusters I and II accounted for $51 \%$ of the specimens.

As a general rule, the highest anisotropy values were obtained for total anisotropy, which means that anisotropy is more accentuated in one spatial direction due, perhaps, to a slight laminar orientation not perceptible to the naked eye.

Sonic velocity clearly declined after the salt crystallization cycles, much more steeply in the Redueña (up to 67\%) than in the Torrelaguna (up to 29\%) dolostone. The specimens with the sharpest decline in ultrasonic propagation velocity exhibited greater deterioration, while anisotropy was found to rise more in the cluster I specimens, i.e., the lowest anisotropy group. This may be interpreted to mean that in initially scantly anisotropic materials, deterioration may induce greater anisotropy. The relative anisotropy values $(\mathrm{dm})$ were less clearly patterned than the total anisotropy data. As Figure 3 shows, in this type of stone, salt crystallization generates scaling, cracks and spalling parallel to the surface of the side of the cube, which would explain the increase in anisotropy.

The total anisotropy index, $d M$, appeared to follow the same upward pattern in the Torrelaguna dolostone, although the variations were smaller. In this case, however, the results were too scattered to assert that the greatest variations were found in specimens with initially lower anisotropy. This stone exhibited a rise in the relative anisotropy index that was not observed in the Redueña material.

Of the two varieties, weight loss was much greater in Redueña, particularly bearing in mind that this dolostone underwent seven ageing cycles compared to the 
ciclos y la de Torrelaguna, 15. Igualmente, el aumento de la absorción de agua y de la porosidad es bastante más significativo para Redueña que para Torrelaguna.

En cuanto a las curvas de distribución porosimétrica, para Redueña se observa que la porosidad accesible al mercurio aumenta después de los ciclos de cristalización de sales, siendo mayor para las probetas de menor grado de anisotropía. En el caso de la muestra de menor anisotropía, el volumen de poros con diámetro entre 10 y $20 \mu \mathrm{m}$ aumenta considerablemente respecto al volumen inicial, mientras que se reduce el porcentaje de poros con diámetro comprendido entre 1 y $3 \mu \mathrm{m}$. Prácticamente el mismo patrón se produce para la muestra de mayor anisotropía, con un incremento mucho más espectacular de la población de poros de 10-20 $\mu \mathrm{m}$. La interpretación estaría relacionada con la generación de las escamas y microescamas.

Respecto a la dolomía de Torrelaguna, el patrón de distribución de tamaño de poro con el envejecimiento es similar (Figura 4). Las muestras envejecidas también experimentan un aumento, aunque menor que en Redueña, de la porosidad accesible al mercurio, que es más alta para las probetas de menor anisotropía. Sin embargo, la distribución porosimétrica que se obtiene de las muestras, tanto la de mayor como la de menor anisotropía, es una disminución de la población de poros entre 1 y $2 \mu \mathrm{m}$, y un ligero aumento de la población entre 0,1 y $1 \mu \mathrm{m}$. Se interpreta este hecho como que un menor grado de deterioro en la dolomía de Torrelaguna, implica un menor cambio en la distribución de tamaño de poros y que las formas de alteración son diferentes en ámbos tipos de piedras.

\section{CONCLUSIONES}

La calidad de la dolomía de Torrelaguna, inicialmente superior a la de Redueña, se traduce en una mejor respuesta frente al deterioro, en este caso concreto, frente a los ciclos de cristalización de sales. Exactamente lo contrario puede decirse de la dolomía de Redueña, que con mayor porosidad y absorción de agua, menor velocidad sónica y características petrográficas y texturales menos favorables, se deteriora de manera mucho más intensa, y en menor tiempo.

Respecto a la relación de las características y propiedades determinadas con respecto a los índices de anisotropía calculados, la relación no es tan clara. Las variedades pétreas presentan una anisotropía moderada-baja, por lo general es mayor la anisotropía total que la relativa, con una importante dispersión de resultados, aunque las poblaciones o clusters determinados de menor anisotropía son los más representativos. La tendencia lógica de que a medida que aumenta la anisotropía disminuye la velocidad sónica, sólo se cumple para Redueña.
Torrelaguna stone's fifteen. Similarly, water absorption and porosity increased more significantly in the Redueña than in the Torrelaguna dolostone.

The pore size distribution curves showed that in the Redueña variety, mercury-accessible porosity increased after salt crystallization to a greater degree in specimens with lower anisotropy. In the sample with lowest anisotropy, the volume of pores with diameters ranging from 10 to $20 \mu \mathrm{m}$ rose substantially with respect to the initial volume, while the percentage of pores with diameters of between 1 and $3 \mu \mathrm{m}$ declined. Practically the same pattern was observed in the sample with the highest anisotropy, although here the increase in the population of 1020- $\mu$ m pores was much steeper. This finding should be interpreted in the context of the generation of scales and microscales.

A similar pattern of change in the pore size distribution with ageing was found for the Torrelaguna dolostone (Figure 4). The aged samples also showed an increase in mercury-accessible porosity, higher for the samples exhibiting lower anisotropy, but smaller in any event than in the Redueña material. The pore size distribution obtained in both low and high anisotropy samples, however, showed a decline in the population of 1 to $2-\mu \mathrm{m}$ pores and a slight increase in the pores measuring 0.1 to $1 \mu \mathrm{m}$. This finding is interpreted to mean that the lower degree of deterioration in the Torrelaguna dolostone entailed a slighter change in pore size distribution and that alteration differed in form in the two types of stone.

\section{CONCLUSIONS}

The initially higher quality of the Torrelaguna dolostone translated into better performance than observed in the Redueña material when exposed to salt crystallization cycles. Indeed, in the Redueña variety, the greater porosity and water absorption, lower sonic velocity and less favourable textural and petrographic characteristics determined much more intense deterioration in less time.

The relationship between stone characteristics and properties and anisotropy indices was less clear. Both varieties showed moderate to low anisotropy, with higher total than relative anisotropy, generally speaking, and widely scattered results. In any event, the populations or clusters with lower anisotropy values were the most representative. The logical pattern of declining sonic velocity with rising anisotropy was only observed for the Redueña variety. 
Las formas de deterioro son distintas para ambos tipos de piedras. La dolomía de Redueña presenta procesos de micro-descamaciones con generación de desplacados, mientras que la piedra de Torrelaguna genera en las probetas morfologías redondeadas por formación de descamados concéntricos. Estas morfologías de deterioro se aprecian en la cara superior de las probetas por lo que existe un condicionante en la orientación de las probetas en las cámaras de ensayo.

El deterioro tras los ciclos genera un aumento de la porosidad (accesible al agua y al mercurio) y de la absorción de agua, y, por tanto, una disminución de la velocidad de transmisión ultrasónica. Las probetas con una mayor disminución de la velocidad de propagación de ultrasonidos son en las que se observan mayores pérdidas de material. Pero no ha sido posible establecer una relación clara entre intensidad de deterioro e índices de anisotropía iniciales. En general, se observa un incremento del índice de anisotropía total, por lo general algo menor para el índice de anisotropía relativa, sin poder apreciar que las variaciones sean mayores en un grupo de anisotropía específico. Sí que es cierto, que para el caso de Redueña las piedras de menor índice de anisotropía total, tienden aumentar de forma más considerable este índice por el deterioro producido por efecto de la cristalización de sales. Se puede apuntar la posibilidad de que materiales inicialmente menos anisótropos puedan aumentar su anisotropía en función de las formas de deterioro que se generan. Pero no se ha observado ninguna relación entre grado de deterioro y grado de anisotropía, o dicho de otra forma, la anisotropía de los materiales pétreos estudiados no parece controlar de forma clara el proceso de su deterioro al ser sometidos a un envejecimiento por cristalización de sales.

Uno de los aspectos más relevantes de este trabajo ha sido el de conocer en detalle el proceso de deterioro de estos materiales. Se trata de dolomías que en muchas ocasiones aparecen de forma conjunta en los edificios, habiéndose observado que el comportamiento frente al mecanismo de cristalización de sales simulado mediante ensayo es bastante distinto, y más drástico, severo y rápido para Redueña que para Torrelaguna. Este hecho nos indica que en casos de restauración futura, en los que haya que realizar intervenciones de sustitución, habría que estudiar este aspecto cuando se mezclasen distintos tipos de materiales pétreos, tanto en obra nueva como patrimonial, ya, que, como se ha determinado en este trabajo, no se sugiere la combinación de ambos materiales, por el deterioro diferencial que se pudiera generar.

\section{AGRADECIMIENTOS}

Nuestro agradecimiento a la Comunidad de Madrid por la financiación obtenida a través del proyecto MATERNAS
The forms adopted by deterioration differed in the two types of stone. Redueña dolostone exhibited microscaling with ultimate spalling, while the Torrelaguna stone generated rounded morphologies in the specimens due to the formation of concentric scaling. These forms of deterioration appeared at the top of the specimens, indicating the existence of a conditioning factor in connection with specimen orientation in the test chambers.

Post-test cycle deterioration generated an increase in (water- and mercury-accessible) porosity and water absorption and, therefore, a decline in ultrasonic transmission velocity. The specimens with the greatest decline in ultrasonic propagation velocity exhibited the greatest dimensional loss. But no clear relationship could be established between intensity of deterioration and initial anisotropy indices. As a general rule, total anisotropy was observed to increase more than relative anisotropy, although these variations were not found to be more intense in any given anisotropic cluster. What was observed in the Redueña stone was that in specimens with lower total anisotropy, this index tended to rise more substantially due to the deterioration induced by salt crystallization. The increase in anisotropy in materials that are initially less anisotropic may depend on the form that deterioration adopts. Nonetheless, no relationship was observed between degree of deterioration and degree of anisotropy; in other words, in the materials studied, anisotropy did not appear to clearly govern the deterioration process induced by salt crystallization ageing.

One of the most relevant aspects of this study was the acquisition of a detailed understanding of deterioration in these materials. The Redueña variety has been found to react very differently -more drastically, severely and rapidly - to salt crystallization ageing than the Torrelaguna stone. These dolostones have sometimes been used in combination in construction. The inference is that in future restoration work involving stone replacement, this aspect should be studied when mixing different types of materials, both in new and heritage buildings for, as this paper shows, combinations of the two are not recommended due to the differential deterioration that may be generated.

\section{ACKNOWLEDGEMENTS}

This study was funded by the Government of the Community of Madrid under the MATERNAS project 
(Durabilidad y conservación de materiales tradicionales naturales del patrimonio arquitectónico (MATERNAS CM 0505/MAT/0094) Este trabajo forma parte del Programa Consolider-Ingenio 2010 (CSD2007-0058), al programa Ramón y Cajal del Ministerio español de Educación, y a la Unión Europea por el proyecto Marie Curie (Advanced research training on the conservation of cultural heritage, MEST-CT-2004-513915).
(Durability and conservation of traditional natural materials in heritage architecture) (MATERNAS CM 0505/MAT/0094); the Spanish Ministry of Education as part of the Consolider-Ingenio 2010 programme (CSD2007-0058) and Ramón y Cajal programme; and the European Union under the Marie Curie project (Advanced research training on the conservation of cultural heritage, MEST-CT-2004-513915).

\section{BIBLIOGRAFÍA / BIBLIOGRAPHY}

(1) Sousa, L.M.O., Suarez del Río, L. M., Calleja, L. Ruiz de Argandoña, V.G., Rodríguez Rey, A.: "Influence of microfractures and porosity on the physico-mechanical properties and weathering of ornamental granites". Eng Geol, 77 (2005), pp. 153-168.

(2) Ruedrich, J., Siegesmund, S.: "Salt and ice crystallisation in porous sandstones". Environ Geol, Vol. 52 (2007), pp. 225-249.

(3) Prrikryl, R.: "Some microstructural aspects of strength variation in rocks". Int J Rock Mech \& Min Scien, Vol. 38 (2001), pp. $671-682$.

(4) Álvarez de Buergo, M., González, T.: "Estudio del método de la medida de la velocidad de propagación del sonido y su aplicación a edificios históricos". Ingeniería Civil, Vol. 94 (1994), pp. 69-74.

(5) Cazalla, O., Sebastián, E., Cultrone, G., Nechar, M., Bagur, M. G.: "Three-way ANOVA interaction analysis and ultrasonic testing to evaluate air lime mortars used in cultural heritage conservation projects". Cem Concr Res, Vol. 29 (1999), pp. 1749-1752.

(6) Dapena, E., Ordóñez, S., García del Cura, M. A.: "Estudio de las rocas calizas utilizadas durante los siglos XVIII y XIX en la construcción de los palacios de Madrid". Ingeniería Civil, Vol. 71 (1989), pp. 67-77.

(7) Zezza, U.: "Physical-mechanical properties of quarry and building stones", In: Advanced Workshop: Analytical Methodologies for the Investigation of Damaged Stone, Veniale, F., Zezza, U. (Eds.), Pavia University, Pavia, Italy, (1990).

(8) Fort, R., Mingarro, F., Lopez de Azcona, M. C.: "Petrología de los materiales de construcción del Palacio Real de Madrid". Geogaceta, Vol. 20, no 5 (1996), pp. 1236-1239.

(9) Fort, R., Bernabeu, A., García del Cura, M. A., López de Azcona, M. C., Ordóñez, S., Mingarro, F.: "Novelda stone: widely used within the Spanish architectural heritage". Mater. Construcc. Vol. 52, no 266 (2002), pp. 19-32.

(10) Přikryl, R., Lokajíčekb, T., Prosa, Z., Klímab, K.: "Fabric symmetry of low anisotropic rocks inferred from ultrasonic sounding: Implications for the geomechanical models". Tectonophysics, Vol. 431, no 1-4 (2007), pp. 83-96.

(11) Del Río, L. M., Lopez, F., Esteban, F. J., Tejado, J. J., Mota, M. I., Gonzalez, I., San Emeterio, J. L. Ramos, A.: "Ultrasonic characterization of granites obtained from industrial quarries of Extremadura (Spain)". Ultrasonics, Vol. 44, Supplement 1 (2006), e1057-e1061.

(12) Buj, O., Gisbert, J.: "Petropysical characterization of three commercial varieties of miocene sandstones from the Ebro Valley". Mater Construcc., Vol. 57, no 287 (2007), pp. 63-74.

(13) Weiss, T., Siegesmund, S., Rosolofosaon, P.: "The relationship between deterioration, fabric, velocity and porosity constraint". In: 9th International Congress on deterioration and conservation of stone, Fassina V, (ed.), Vol. 1, 215-223 (2000). Elsevier.

(14) Fort, R., Rodríguez, J.: "Prospección ultrasónica para determinar el deterioro de la Estela de Barros (Cantabria)", III Congreso Nacional de Arqueometría (2003), 51-57, ISBN: 84-472-0552-5.

(15) Ruiz de Argandoña, V. G., Calleja, L., Suárez del Río, L. M., Rodríguez-Rey, A., Velorio, C.: "Durabilidad en ambientes húmedos de la Arenisca de la Marina (Formación Lastres, Jurásico Superior de Asturias)". Trabajos de Geología, Universidad de Oviedo, Vol. 25 (2005), pp. $105-115$.

(16) Yavuz, H., Altindag, R., Sarac, S., Ugur, I., Sengun, N.: "Estimating the index properties of deteriorated carbonate rocks due to freeze-thaw and thermal shock weathering". Int J Rock Mech \& Min Ssci, Vol. 43 (2006), 767-775.

(17) Esbert, R. M.: "Alteration of granite stone used in building construction" Mater Construcc., Vol. 58, no 288 (2007), 77-89.

(18) Sebastian, E., de la Torre, M. J., Cazalla, O., Cultrone, G., Rodriguez-Navarro, C.: "Evaluation of treatments on biocalcarenites with ultrasound". The e-Journal of non-destructive testing, Vol. 4, no 12 (1999).

(19) Myrin, M., Malaga, K.: "A case study on the evaluation of consolidation treatments of Gotland sandstone by use of ultrasound pulse velocity measurements". In: Heritage, Weathering and Conservation. Ed. Fort, R., Alvarez de Buergo, M., Gomez-Heras, M., VazquezCalvo, C., Taylor \& Francis Group, London, (2006), pp. 749-755.

(20) Dreesen, R., Dusar, M.: "Historical building stones in the province of Limburg (NE Belgium): role of petrography in provenance and durability assessment". Mater. Charact. Vol. 53, no 2-4 (2004), pp. 273-287.

(21) Gomez-Heras, M., Fort, R.: "Location of quarries of non traditional stony materials in the architecture of Madrid: the Crypt of Catedral of Santa María la Real de la Almudena". Mater. Construcc. Vol. 54, nº 274 (2004), pp. 33-48.

(22) Galán, E., Carretero, M. I., Mayoral, E.: "A methodology for locating the original quarries used for constructing historical buildings: application to Málaga Cathedral, Spain". Eng. Geol. Vol. 54 (1999), pp. 287-298.

(23) Birch, F.: "The velocity of compressional waves in rocks to 10 kilobars, part 2". J Geophys. Res. Vol. 66 (1961), $2199-2224$.

(24) Guydader, J., Denis, A.: "Propagation des ondes dans les roches anisotropes sous contrainte évaluation de la qualité des schistes ardoisiers". Bull. Eng. Geo. Vol. 33 (1986), pp. 49-55. 
(25) Sheremeti-Kabashi, F., Snethlage, R.: "Determination of structural anisotropy of Carrara Marble with ultrasonic measurements". In: 9th International Congress on Deterioration and Conservation of stone, V. Fassina V (ed.), Vol. 1 (2000), pp. 247-253, Elsevier.

(26) Fort, R., Varas, M. J., Pérez-Monserrat, E. M., Luque, J., Alvarez de Buergo, M., Vazquez-Calvo, C.: "Los ladrillos del recinto amurallado de Talamanca de Jarama, Madrid: criterios para su diferenciación". Bol. Soc. Esp. Ceram. V, Vol. 46 (2007), pp. 145-152.

(27) Schön, J. H.: "Physical properties of rocks. Fundamentals and principles of petrophysics". Tarrytown, New York, Pergamon Press, 583 p. (1996).

(28) Correns. C. W.: "Growth and dissolution of crystals under linear pressure". Disc Faraday Soc. Vol. 5 (1949): pp. $267-271$.

(29) Arnold, A. \& Zehnder, K.: "Salt Weathering on monuments". In Conservation of Monuments in the Mediterranean Basin, F. Zezza, ed. Grafo, Brescia, 31-58. (1990).

(30) Price. C. A.: Stone Conservation: "An Overview of Current Research". The Getty Conservation Institute, Sta. Monica, 88 p., (2006).

(31) Goudie, A., Viles, H.: "Salt Weathering Hazards". 235 p., Wiley, Chichester, (1997).

(32) Benavente, D., García del Cura, M. A., Fort, R., Ordóñez, S.: "Thermodynamic modelling of changes induced by salt pressure crystallisation in porous media of stone". J Cryst.Growth. Vol. 204 (1999), pp. 168-178.

(33) Rodriguez-Navarro, C., Dohene, E.: "Salt weathering: influence of evaporation rate, supersaturation and crystallisation pattern". Earth Surf Proc Land, Vol. 24 (1999), pp. 191-209.

(34) Scherer, G. W.: "Crystallisation in pores". Cem. Concr. Res. Vol. 29 (1999), pp. 1347-1358.

(35) Gomez-Heras, M., Benavente, D., Alvarez de Buergo, M., Fort, R.: "Soluble salt minerals from pigeon droppings as potential contributors to the decay of stone based Cultural Heritage". Eur. J Mineral. Vol. 16 (2004), pp. 505-509.

(36) Gomez-Heras, M., Fort, R.: "Patterns of halite ( $\mathrm{NaCl}$ ) crystallisation in building stone conditioned by laboratory heating regimes". Environ. Geol. Vol. 52 (2007): pp. 239-247.

(37) UNE-EN 12370: 1999, "Métodos de ensayo para piedra natural. Determinación de resistencia a la cristalización de sales/ Natural stone test methods - Determination of resistance to salt crystallisation", AENOR, (1999).

(38) RILEM: "Recommended tests to measure the deterioration of stone and to assess the effectiveness of treatment methods". Mater. et Const. Vol. 13 (1980), pp. 175-253.

(39) Alonso, F. J., Ordaz, J., Valdeón, L., Esbert, R.: "Revisión crítica del ensayo de cristalización de sales". Mater. Construcc. Vol. 206 (1987), pp. 53-60.

(40) Benavente, D., García del Cura, M.A., Bernabeu, A., Ordóñez, S.: "Quantification of salt weathering in porous stones using an experimental continuous partial immersion method". Eng. Geol. Vol. 59 (2001), pp. 313-325.

(41) Tsui, N. Flatt, R. J., Scherer, G. W.: "Crystallization damage by sodium sulfate". J Cult. Herit. Vol. 4 (2003), 109-115.

(42) Mohammad, B. K.: "The salt durability of some Jordanian limestones as a function of their petrophysical properties". The Electronic Journal of Geotechnical Engineering, Vol. 8 (2003) http://www.ejge.com/2003/Ppr0312/Ppr0312.htm

(43) Angeli, M., Bigas, J. P., Benavente, D., Menendez, B., Hebert, R., David, C.: "Salt crystallization in pores: quantification and estimation of damage". Environ Geol, Vol. 52 (2007), pp. 205-213.

(44) Menduiña, J., Fort, R., García del Cura, M. A., Galán, L., Pérez-Soba, C., Perez-Monserrat, E. M., Fernández-Revuelta, B., Bernabéu, A., Varas, M. J.: "Las piedras utilizadas en la construcción de los bienes de interés cultural de la Comunidad de Madrid anteriores al siglo XIX", Instituto Geológico y Minero de España. (2005). 131 p.

(45) Evamy, B.D.: "The application of a chemical staining technique to a study of dedolomitisation". Sedimentology, Vol. 2 (1963), 164-170. (46) UNE-EN 14579: 2005, Métodos de ensayo para piedra natural, Determinación de la velocidad de propagación de los ultrasonidos/ Natural stone test methods - Determination of sound speed propagation, AENOR, (2005).

(47) Khan, S. S., Ahmad, A.: "Cluster center initialization algorithm for k-means clustering". Pattern. Recogn. Lett. Vol. 25 (2004), pp. 12931302.

(48) UNE-EN 1936:1990, Métodos de ensayo para piedra natural, Determinación de la densidad real, aparente, la porosidad abierta y total/ Natural stone test methods. Determination of real density and apparent density and of total and open porosity, AENOR, (1990).

(49) UNE-EN 13755:2004, Métodos de ensayo para piedra natural, Determinación del coeficiente de absorción de agua por presión atmosférica/ Natural stone test methods. Determination of water absorption at atmospheric pressure, AENOR, (2004).

(50) Ordóñez, S., Fort, R., García del Cura, M. A.: "Pore size distribution and the durability of a porous limestone". Q J Engin. Geol. Vol. 30, no 3 (1997), pp. 221-230. 